\title{
Trastornos fisiológicos de la papa - Necrosis por calor interno $^{1}$
}

\author{
L. Zotarelli, J. E. Reyes-Cabrera, C. M. Worthington, C. Hutchinson, S. Byrd, D. Gergela, y D. L. \\ Rowland ${ }^{2}$
}

En el Norte de la Florida, la siembra de papa comienza a finales de diciembre cuando la duración del día es corto y la temperatura del suelo es relativamente fresca. A medida que la temporada avanza, las horas de luz del día y la temperatura del aire aumentan como la planta de papa llega a sus etapas claves de desarrollo (emergencia, crecimiento vegetativo, la iniciación del tubérculo). En Florida las papas son típicamente cultivadas en los suelos arenosos de textura gruesa, con baja capacidad de retención de agua y con alto potencial de lixiviación de nutrientes.

Las potenciales precipitaciones fuertes durante la etapa de crecimiento pueden causar la lixiviación de nutrientes móviles como el nitrato-N, resultando a menudo en un estrés nutricional para la planta de papa y la iniciación de defectos fisiológicos, como son el centro marrón, el corazón hueco y la necrosis por calor interno.

La necrosis por calor interno (NCI) es un trastorno fisiológico que causa un pardeamiento inaceptable del tejido del tubérculo y puede causar pérdidas económicas para el productor (Stevenson et al. 2001). Las tres principales causas de la NCI en los tubérculos es la alta temperatura en el suelo, la humedad inadecuada del suelo y la nutrición sub-óptima de la planta, o la combinación de estos factores.
La NCI se describe como un trastorno fisiológico causado por las temperaturas elevadas durante las últimas etapas de desarrollo y crecimiento del tubérculo (Stevenson et al. 2001). La NCI es más común en los suelos arenosos (O’Brien y Rich 1976). Ésta puede ser especialmente severa y los síntomas pueden empeorar a medida que la temporada de crecimiento progresa (Stevenson et al. 2001). Los tubérculos que crecen cerca de la superficie del suelo pueden experimentar altas temperaturas antes de la muerte del follaje o de la cosecha y por lo tanto son muy susceptibles al desarrollo de la enfermedad (Hardy 1996; Stevenson et al. 2001). Si el follaje se encuentra verde y activamente creciendo durante este período de temperaturas elevadas, el agua y los nutrientes se traslocan fuera del tubérculo para abastecer la planta. Esta exportación masiva de agua y nutrientes crea un estrés en el sistema vascular dentro del tubérculo hasta que el anillo vascular eventualmente se deteriora y se vuelve necrótico. Esto conduce a la conversión oxidativa de fenólicos orgánicos a quinonas en el tejido del tubérculo y da como resultado el pardeamiento del tejido interno del tubérculo.

Niveles bajos de calcio pueden jugar un papel importante en la ocurrencia de NCI. Varios estudios han encontrado una relación entre los niveles bajas de calcio en los tubérculos y el aumento de la incidencia de NCI (Davies 1998;

1. Este documento, HS1221, es uno de una serie de publicaciones del Departamento del Ciencias para la Horticultura, Servicio de Extensión Cooperativa de la Florida, Instituto de Alimentos y Ciencias Agrícolas, Universidad de la Florida. (UF/IFAS). Fecha de primera publicación: mayo 2013. Visite nuestro sitio web EDIS en http://edis.ifas.ufl.edu.

2. L. Zotarelli, profesor asistente; Joel E. Reyes-Cabrera, estudiante graduado; Christine M. Worthington, estudiante graduado; y C. Hutchinson, profesor asociado, Departamento de Ciencias Hortícolas; S. Byrd, el estudiante graduado, Departamento de Agronomía, D. Gergela, coordinador de la investigación, Alianza para la Sustentabilidad del Agua en Florida, Agricultura y Comunidad en Hastings, y Rowland DL, profesor asociado de la Facultad de Agronomía, Instituto de Alimentos y Ciencias Agrícolas, Universidad de la Florida, Gainesville, FL 32611. 
Sterrett y Henninger 1991). El calcio es transportado a los tubérculos a través del floema, mientras que las hojas lo reciben a través del xilema. El xilema es la vía de transporte principal para el calcio dentro de la planta. Por lo tanto, el contenido de calcio es a menudo mayor en las hojas que en el tubérculo (Davies 1998), lo que posiblemente contribuye al desarrollo de NCI.

La baja humedad del suelo también podría influir en la ocurrencia de la IHN, ya que disminuiría el uso total de agua en la planta, pot lo tanto restringiendo el transporte de calcio (Stevenson et al. 2001).

En los tubérculos, las áreas necróticas son mayormente encontradas en/alrededor del anillo vascular, usualmente juntas y apuntando hacia el centro (médula). (Figura 1). En algunas variedades, síntomas pueden ser detectados en el exterior del tubérculo con necrosis interna, pero esto no es siempre el caso, ya que algunas variedades nunca presentan síntomas exteriores visibles (por ejemplo papas tipo 'Atlántico') (Sterrett, Henninger, and Lee 1991).

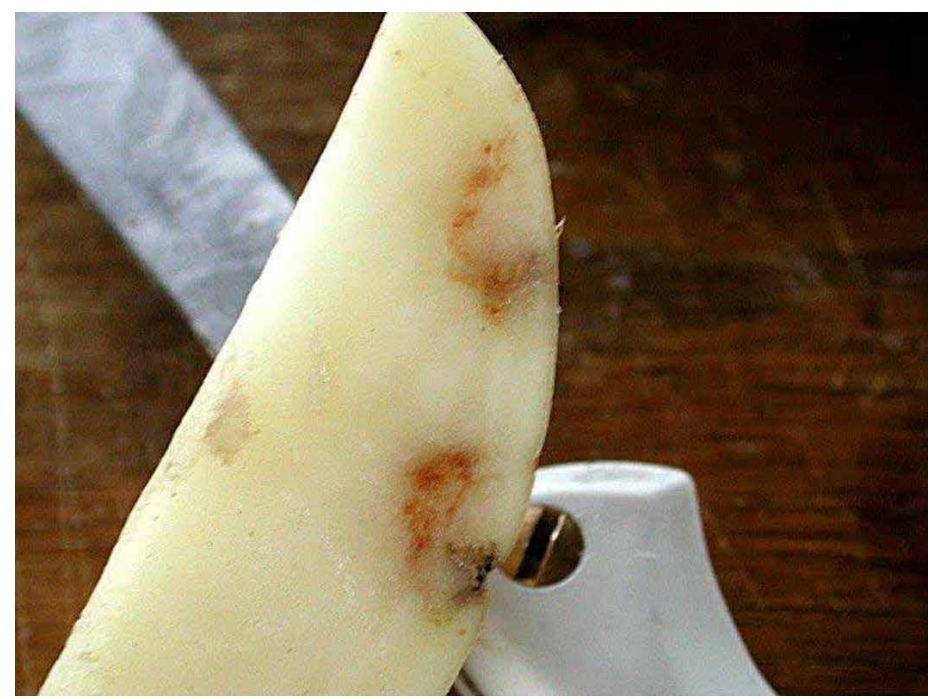

Figura 1. Los síntomas de necrosis de calor interno en el mercado fresco de papas 'Red LaSoda'.

Créditos: Chad Hutchinson

Atlántico es la principal variedad para papa frita cultivada en el norte de Florida, por lo que es económicamente vital para la zona. Los mayores procesadores de papas fritas solicitan 'Atlantico' por sus cualidades de procesamiento, como son, el color claro de la papa frita, relativo alto rendimiento y alta densidad relativa (Webb et al. 1978). Sin embargo, 'Atlantico' es susceptible a NCI. En un estudio por Henninger, Sterrett, y Haynes (2000) en la relación entre NCI y las características de la papa, los tubérculos más grandes experimentaron una mayor ocurrencia y severidad del trastorno. De las 19 variedades estudiadas, 'Atlantico' fue encontrada la variedad con mayor inclinación al desarrollo del trastorno y tuvo los casos más severos de NCI (Henninger, Sterrett, y Haynes 2000).

La necrosis interna también ha sido llamada la mancha interna marrón (IBS por sus iniciales en ingles), la mancha de color chocolate o mancha de óxido (Sterrett y Henninger 1997). Aunque tienen varias similitudes, la necrosis por calor interno y la mancha interna marrón son reconocidas como dos trastornos diferentes (Christ 1998; Stevenson et al. 2001). A diferencia de IBS, la cual se ha reportado que ocurre durante la temporada de crecimiento, NCI en 'Atlántico' ha sido reportada ocurriendo entre la mitad y el final de período de llenado del tubérculo. Worthington et al. (2006) demostró que la NCI en la producción de papas en Florida es desencadenada por eventos de precipitación fuerte que pueden resultar en la lixiviación de nutrientes y condiciones nutricionales que estresan la planta temprano en la temporada, en conjunto con una temperatura diaria mínima relativamente alta al final de la temporada (Yencho et al. 2008).

En el 2003, la variedad 'Harley Blackwell' fue liberada al mercado por el USDA, proporcionando a los productores de papas fritas una alternativa a la papa 'Atlántico'. Durante 12 temporadas de evaluación, el rendimiento comercial de 'Harley Blackwell' fue de 6\% más bajo que 'Atlántico' (287 y 304 quintales por acres ${ }^{-1}$ respectivamente). Adicionalmente, la densidad específica de 'Harley Blackwell' (1.075) fue ligeramente inferior que la de 'Atlántico' (1.081), pero aún un valor aceptable según los estándares para procesamiento de papas fritas (BARC/USDA 2004; USDA 1978). 'Harley Blackwell' fue resistente a NCI como también al corazón hueco (Sterrett 2009). Otros clones resistentes a la NCI son actualmente evaluados por la 'Water Agriculture and Community Sustainability' en Hastings, Florida (2010) demostró que la variedad 'Snowden' and 'Elkton' también tiene una baja incidencia de NCI, así como corazón hueco y centro marrón, en comparación con 'Atlántico.' 'Snowden' también obtuvo un mayor rendimiento total y comercial, así como también una densidad relativa más alta que 'Atlántico' (“Chipping Potato Variety Trial” 2010).

Aunque las condiciones climáticas adversas no pueden ser evitadas durante la temporada de crecimiento, los productores tienen opciones que pueden ayudar a reducir la incidencia de los tubérculos con NCI. El balance de los nutrientes debe ser manejado adecuadamente para reducir el estrés de la planta al comienzo de la temporada, particularmente durante el crecimiento rápido y el desarrollo de la planta. Esto es especialmente necesario para el calcio, el cual debe ser suministrado a la profundidad en la cual el desarrollo de las raíces y tubérculos ocurren (Stevenson et 
al. 2001). El cultivo de clones resistentes a la NCI también ayuda a reducir la incidencia de la NCI.

La variedad 'Harley Blackwell' se puede usar para plantaciones tardías cuando las condiciones prevalentes son más cálidas y húmedas y el desarrollo de NCI en 'Atlántico' es más alto. El prevenir que los tubérculos poco profundos se expongan también ayuda a prevenir la ocurrencia de la NCI, mientras que el riego en cantidades óptimas y el aumento de una cubierta del suelo puede reducir el exceso de temperatura que experimentan los tubérculos a poca profundidad (Stevenson et al. 2001). Sin embargo, si el riego se está usando, la humedad del suelo debe ser cuidadosamente monitoreada para que condiciones favorables para otros trastornos o enfermedades no se promuevan (Stevenson et al. 2001). Stevenson et al. (2001) también indica que la presencia de una cubierta vegetativa adecuada es beneficial para la reducción de la incidencia de la IHN, quizás debido al efecto sombra y el descenso de la temperatura del suelo sobre los tubérculos. Por último, la cosecha de los tubérculos para evitar o minimizar la exposición a altas temperaturas y guardar los tubérculos en condiciones apropiadas son también métodos para ayudar a reducir la incidencia de NCI (Stevenson et al. 2001).

\section{Recursos Adicionales}

Worthington, C. M. 2006. "Timing of Climatic Factors that May Influence Potato Yield, Quality, and Potential Nitrogen Losses in a Northeast Florida Seepage-Irrigated Potato Production System." Ph.D. diss. Gainesville: University of Florida.

Zotarelli, L., P. D. Roberts, W. M. Stall, S. E. Webb, S. A. Smith, B. M. Santos, S. M. Olson, and E. H. Simonne. 2011. "Potato Production in Florida." In Vegetable Production Handbook for Florida 2010-2011, 243-259. Gainesville: University of Florida Institute of Food and Agricultural Sciences

\section{Referencias}

Beltsville Agricultural Research Center (BARC/USDA). 2004. "Vegetable Lab News." http://www.ba.ars.usda.gov/ psi/vl/vlnews.htm\#Harley_Blackwell.

“Chipping Potato Variety Trial." 2010. In Florida Potato Variety Trial, 2010, 173-176. Gainesville: University of Florida Institute of Food and Agricultural Sciences. http:// hastings.ifas.ufl.edu/research_projects/2010_potato_variety_trial_report/chapter_11.pdf.
Christ, B. J. 1998. "Identifying Potato Diseases in Pennsylvania." Penn State College of Agricultural Sciences. Accessed January 11, 2012. http://pubs.cas.psu.edu/FreePubs/ pdfs/agrs75.pdf.

Davies, H. V. 1998. "Physiological Mechanisms Associated with the Development of Internal Necrotic Disorders of Potato." Amer. J of Potato Res. 751: 37-44.

Hardy, B., ed. 1996. Major Potato Diseases, Insects, and Nematodes. Lima, Peru: International Potato Center.

Henninger, M. R., S. B. Sterrett, and K. G. Haynes. 2000. "Broad-Sense Heritability and Stability of Internal Heat Necrosis and Specific Gravity in Tetraploid Potatoes." Crop Sci. 40 (4): 977-984.

O’Brien, M. J., and A. E. Rich. 1976. "Potato Diseases." In USDA Agriculture Handbook (No. 474). Washington, DC: U. S. Department of Agriculture. http://naldc.nal.usda.gov/ download/CAT31007970/PDF.

Sterrett, R. 2009. Harley Blackwell - A Recent Potato Release from USDA. Blacksburg: Virginia Cooperative Extension.

Sterrett, S. B., and M. R. Henninger. 1991. "Influence of Calcium on Internal Heat Necrosis of Atlantic Potato." Am. Potato J. 68 (7): 467-477.

Sterrett, S. B., and M. R. Henninger. 1997. "Internal Heat Necrosis in the Mid-Atlantic Region - Influence of Environment and Cultural Management." Am. Potato J. 74 (4): 233-243.

Sterrett, S. B., M. R. Henninger, and G. S. Lee. 1991. "Relationship of Internal Heat Necrosis of Potato to Time and Temperature after Planting." J. Amer. Soc. Hort. Sci. 116 (4): 697-700.

Stevenson, W. R., R. Loria, G. D. Franc, and D. P. Weingartner. 2001. "Physiological Disorders of Tubers: Internal Symptoms." In Compendium of Potato Diseases (2nd ed.) St. Paul, MN: American Phytopathological Society.

United States Department of Agriculture (USDA). 1978. "United States Standards for Grades of Potatoes for Chipping." http://www.ams.usda.gov/AMSv1.0/getfile?dDocNa me=STELPRDC5050437.

Webb, R. E., D. R. Wilson, J. R. Shumaker, B. Graves, M. R. Henninger, J. Watts, J. A. Frank, and H. J. Murphy. 1978. "Atlantic: A New Potato Variety with High Solids, Good 
Processing Quality, and Resistance to Pests." Amer. Potato J. 55 (3): 141-145.

Worthington, C. M., K. M. Portier, J. M. White, R. S. Mylavarapu, T. A. Obreza, W. M. Stall, and C. M. Hutchinson. 2007. "Potato (Solanum tubersoum L.) Yield and Internal Heat Necrosis Incidence under Controlled Release and Soluble Nitrogen Sources and Leaching Irrigation Events." Amer. J. of Potato Res. 84 (5): 403-413.

Yencho, G. C., P. H. McCord, K. G. Haynes, and S. B. Sterrett. 2008. "Internal Heat Necrosis of Potato - A Review."

Amer. J. of Potato Res. 85 (1): 69-76. 